\title{
Mesoscopic Finite Element Simulation on the Interfacial Bonding Performance of Functionally Gradient Concrete
}

\author{
Jianmin Wang, ${ }^{1, *}$ Yitao Fan, ${ }^{1}$ Chengfeng Zhu, ${ }^{1}$ Shuang $\mathrm{Lu}^{2}$ and Junzhe $\mathrm{Liu}^{3}$
}

\begin{abstract}
To study the bonding shear performance of the casting interface in the functionally gradient concrete (FGC), a multiphase mesoscopic finite element modeling method was proposed to simulate a sandwich FGC specimen with the normal weight concrete (NWC) cast on the hardened ceramsite lightweight aggregate concrete (LWAC) layer. Four kinds of phases are considered during modeling: coarse aggregates, mortar substrate, interface transition zone (ITZ) between the mortar and coarse aggregates, and the casting interface transition zone (C-ITZ). Each phase in the model is endowed with the respective material property based on the constitutive damage plastic model (CDP). The structural characteristics of the C-ITZ that are related to the casting construction of the FGC are fully considered in the proposed modeling method. Based on the verification by corresponding experiments, the internal damage is developed, and the failure mechanisms of the FGC model are effectively reflected by the simulation. For the designed FGC specimen corresponding to the experiment, the weak position is proved to be located on the C-ITZ. The damage development and damage characteristics of the given FGC specimen are more related to the casting interval time of the FGC.
\end{abstract}

Keywords: Functionally gradient concrete; Mesoscopic finite element; Interfacial bonding performance; Lightweight aggregate. Received:14 March 2020; Accepted: 26 June 2020.

Article type: Research article.

\section{Introduction}

Functionally gradient material (FGM) is a new type of heterogeneous composite material, in which two or more different kinds of materials continuously and smoothly change from one side of the material to the other by advanced composite technology. In 1999, the concept of gradient was first introduced into the interface problem of cement-based concrete; ${ }^{[1]}$ and the research on the $\mathrm{FGC}$ has become an important branch of the engineering material in recent years. ${ }^{[2]}$ The application of the gradient concrete can effectively improve the synthetic performance of cement-based materials to solve the problem of the weaker interface, single functional, lower tensile strength, poor toughness and so on; ${ }^{[3]}$ and it also can extend the applicability of the concrete in complex and special engineering environments. ${ }^{[4]}$ The hybrid FGC,

${ }^{I}$ School of Civil and Environmental Engineering, Ningbo University, 315211, Ningbo, China.

${ }^{2}$ School of Civil Engineering, Harbin Institute of Technology, 150090, Harbin, China.

${ }^{3}$ School of Architecture Engineering, Qingdao Agricultural University. Qingdao, 266109, China.

*E-mail: wangjianmin@nbu.edu.cn (J.Wang); hitlu@126.com (S. Lu). composed of the normal weight concrete (NWC) and the ceramsite lightweight aggregate concrete (LWAC), can effectively integrate the advantages of both types of concrete together. Akmaluddin et al. ${ }^{[5]}$ discussed the connection behavior of the hybrid precast concrete column and the sandwich concrete beam under the static loading. Ji et al.$^{[6]}$ analyzed the variation of the mid-span deflection of the composite beam composed of the reactive powder concrete (RPC) and normal weight concrete (NWC) considering the influence of the prestressing degree, the RPC height and the NWC strength; the higher the pre-stressing degree, the longer the elastic stage before the crack, and the faster the stiffness in strengthening stage decreases after yielding. Campi et al..$^{[7],[8]}$ proposed a closed-form solution on two-layer beams considering the interlayer slip, in which a linear and nonproportional law relating interfacial shear tractions and slips is chosen to describe the interfacial behavior. As a kind of typical multiphase inhomogeneous material, the bonding performance and failure mechanism of FGC need thorough studies from multi perspectives. ${ }^{[9]}$

For the simulation analysis, Yao et al. ${ }^{[9]}$ divided the recycled concrete into five components at the mesoscopic 
scale: old and new hardened mortar units, internal and external interface area units, and natural aggregate units; and established a two-dimensional randomly packing model of circular aggregate. A modeling method of two-dimensional mesoscopic scale random aggregate was proposed to establish the circular, oval, and polygon random aggregate models. ${ }^{[11]}$ Zhang et al. ${ }^{[12]}$ suggested the random walking algorithm for three-dimensional polygonal aggregate packing, and the distribution of aggregates was verified to satisfy the Fuller change curve. In fact, there is a typically physical structure for the interfacial layer in FGC which is important to guarantee the integration and coordination of the members. The structure of the interfacial layer is directly related to the construction technology, the casting interval time of the FGC members and so on. It is needed to carefully consider the physical structure and the material property of the interfacial layer in FGC to accurately simulate the performance of the FGC. In this paper, the sandwich FGC specimen model was designed with NWC cast on the ceramsite lightweight aggregate concrete (LWAC) in layers; and a multiphase mesoscopic finite element modeling method was proposed. The physical structure of the casting interface transition zone (C-ITZ) in FGC was specially modeled in detail and the mechanical performance of the FGC model was simulated based on the experimental results.

\section{Multiphase mesoscopic model of FGC}

\subsection{Structural details of the FGC}

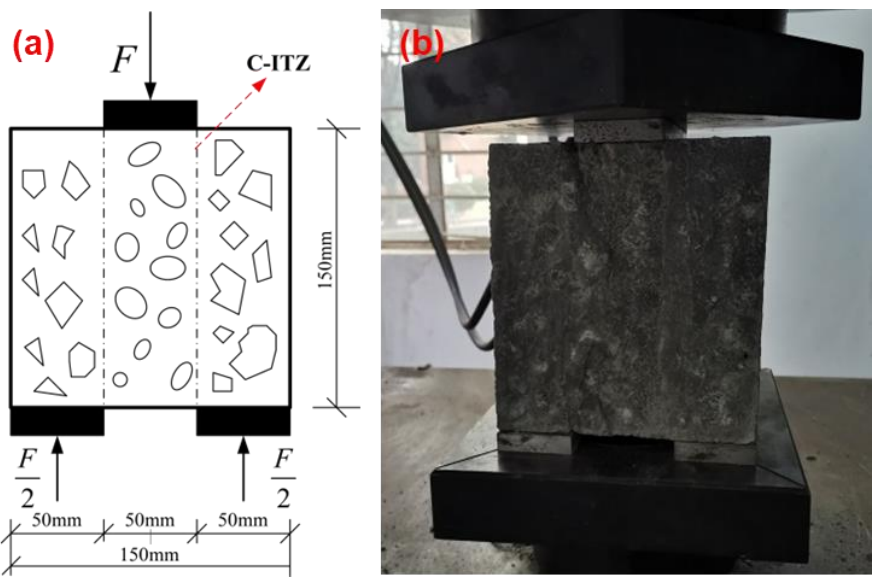

Fig. 1 FGC specimen model and the experiment loading (a) The FGC model; and (b) experiment blocks and loading.

To study the interfacial bonding performance of FGC with NWC cast on the LWAC in layers, a sandwich specimen model is designed as shown in Fig. 1(a). The internal layer is LWAC substrate precast in advance, and two layers on both sides of the middle layer are NWC cast with a certain interval time late. The casting interface of the FGC is handled with the mortar retarder in the experiment. For a single kind of concrete material, it can be simulated at the mesoscopic scale as a composite is composed of the mortar substrate, coarse aggregate, the interface transition zone (ITZ) contacting the mortar and aggregates.
The modeled FGC is considered to be composed of four basic phases due to the special material property and structural detail of the C-ITZ. To describe the properties of the designed FGC model in detail, it can be divided into the normal aggregate (N-AGG), the mortar substrate (N-M) in NWC, ITZ between N-AGG and N-M (N-ITZ), the ceramsite lightweight coarse aggregate (L-AGG), the mortar substrate (L-M) in LWAC, ITZ between L-AGG and L-M (L-ITZ). Besides, the C-ITZ bonding between the NWC and LWAC is an important internal phase in the model.

\subsection{Coarse aggregates}

To build the two-dimensional mesoscopic model of the concrete, the three-dimensional gradation of the coarse aggregate in three-dimension must first be transformed to the two-dimensional. Based on the Fuller formula, the probability transforming the three-dimensional grading curve into that of the two-dimension specimen from a point with the aggregate diameter $D<D_{o}$ is expressed as, ${ }^{[13]}$

$$
\begin{aligned}
& P_{c}\left(D<D_{o}\right)= \\
& P_{k}\left[\begin{array}{c}
1.065\left(\frac{D_{o}}{D_{\max }}\right)^{0.5}-0.053\left(\frac{D_{o}}{D_{\max }}\right)^{4}-0.012\left(\frac{D_{o}}{D_{\max }}\right)^{6} \\
-0.045\left(\frac{D_{o}}{D_{\max }}\right)^{8}+0.0025\left(\frac{D_{o}}{D_{\max }}\right)^{10}
\end{array}\right]
\end{aligned}
$$

in which $D_{0}$ is the sieve diameter, $D_{\max }$ is the maximal aggregate size and $P_{k}$ is the volume ratio of the aggregate to the total volume.

In the two-dimensional model, the probability distribution function $P_{c}\left(D_{o}<D\right)$ is obtained to calculate the amount of coarse aggregates with the diameter equaling to $D_{o}$,

$$
n_{i}=\left(P_{c i}-P_{c i+1}\right) \frac{A}{A_{i}}
$$

where $n_{i}$ denotes the amount of coarse aggregates for a given diameter, $A$ is the total area of the 2-D model, and $A_{i}$ is the cross-sectional area of the considered aggregate.

According to the distribution of particle sizes of the aggregates in the experiment, four gradation parameters are selected to calculate the amount of the ceramsite coarse aggregates and normal coarse aggregates in the FGC model. The sequential aggregate sizes are $2 \mathrm{~mm}-5 \mathrm{~mm}, 5 \mathrm{~mm}-10 \mathrm{~mm}$, $10 \mathrm{~mm}-15 \mathrm{~mm}$ and $15 \mathrm{~mm}-20 \mathrm{~mm}$.

\subsection{Modeling of the FGC}

To build the mesoscopic model of layered FGC, the throwing and locating of coarse aggregates, the building of ITZ surrounding coarse aggregates and C-ITZ between the NWC and LWAC layer are essential. The most important is the modeling of the C-ITZ because it is directly related to the processing method of the casting interface and the constructional procedure. The mortar retarder is used to handle the casting interface of the sandwich FGC blocks in the experiment. The handled interface before casting is shown in 
Fig. 2(a), which characterizes with some of the coarse ceramsite aggregates locally exposed out the casting surface. Therefore, this important structural detail of the casting interface should be characterized in modeling.
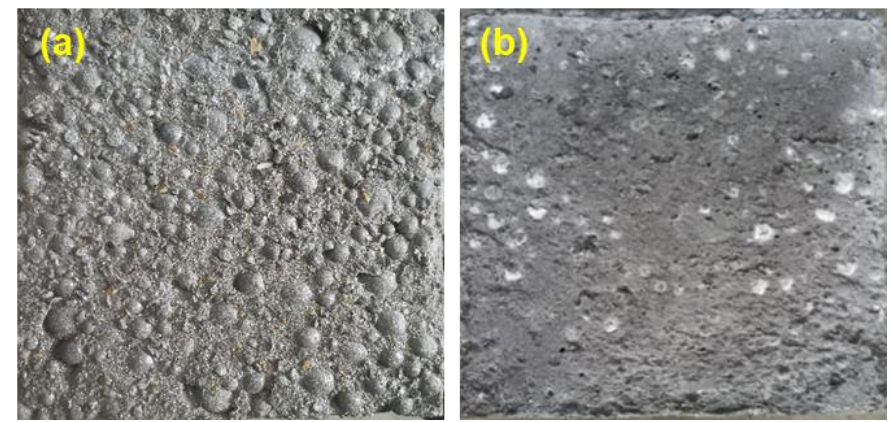

Fig. 2 Appearance of the casting interface ((a) after handled with mortar retarder; and (b) after shear loading experiment).

\subsubsection{Modelling of coarse aggregates and ITZ}

The ceramsite coarse aggregates L-AGG is simplified as the elliptical shape with different sizes and ratios of the long axis to the short during modeling; and the normal coarse aggregates $\mathrm{N}-\mathrm{AGG}$ is simplified as random polygons. The randomly throwing algorithm is adopted to generate the coarse aggregates L-AGG and N-AGG in the model. The circular base is first defined to randomly throw, locate and distinguish the L-AGG and N-AGG in different mortar substrate regions in the model. The grouping, amount and sizes of the circular base are determined according to the gradation of coarse aggregates, the mix design and the volume ratio of aggregates in the experiment. As shown in Fig. 3, the L-AGG is located based on the given random number describing the long axis direction and ratios of the long axis to the short. Similarly, NAGG is generated according to the given side number and vertex coordinates.

The FGC model is a sandwich structure characterized by the LWAC layer clamped by two NWC layers on both sides, as shown in Fig. 4. The middle LWAC layer is first prefabricated; then NWC is cast after a certain interval time and the interface is handled by the mortar retarder. Therefore, the interface handled by the mortar retarder before casting the NWC is characterized with many ceramsite coarse aggregates exposed outside the interface, which is shown in Fig.2(a).

To simulate the structural characteristic of the casting interface in the FGC, the throwing region boundary of L-AGG in LWAC is first given and shown in Fig. 4. Parameter " $a$ " is defined as the thickness of the initial interface handled by the mortar retarder. After the position of L-AGG is finished, the throwing region boundary of the N-AGG in NWC on both sides of the middle LWAC layer is redefined by the casting interface lines. During the throwing, it is needed to ensure that the circular bases cannot collide or contact each other.

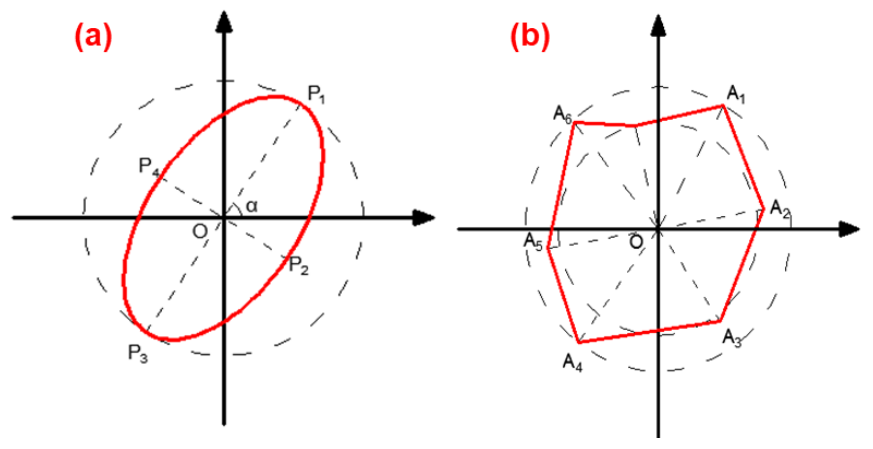

Fig. 3 Circular bases of the aggregate model ((a) L-AGG ceramsite aggregate; (b) N-AGG Polygon aggregate).

The area of all coarse aggregates is about $43 \%$ of the total FGC model area calculated according to Eq. (1) and Eq. (2) from the experimental mixture proportion. Research indicates that the thickness of the ITZ in concrete is about 15 50 microns. ${ }^{[14]}$ The observation results from the scanning electron microscope (SEM) of high-performance concrete indicate that, in the range $(0-100 \mu \mathrm{m})$ of the ITZ, the hydration products, the density of the hydration products and the pores changed as the curing age increased. It can be concluded that the weaker place in the ITZ is at the range of $0-15 \mu \mathrm{m} \cdot{ }^{[15]}$ If the mechanical parameters of each phase in the concrete model are proper, a satisfying simulation result that fits well with the macroscopic mechanical performance can be obtained with a slightly larger thickness of ITZ in the model. ${ }^{[16]}$ Referring to relevant researches and considering the mesh and computing time during the simulating calculation, the thickness of ITZ is assumed as 100 and 50 microns respectively in the given FGC model for comparison. The corresponding parameters setting up in the model are corrected according to the experimental result.

After the initial positioning of L-AGG and N-AGG is finished, the final L-AGG and N-AGG in the model are generated by shrinking the initial L-AGG and N-AGG shapes to a certain ratio based on their circle center. Subtracting the initial L-AGG and N-AGG shapes by their shrunk shapes generates the L-ITZ and N-ITZ model surrounding each coarse aggregate.

\subsubsection{Modelling of C-ITZ}

After building the mortar substrate and L-AGG models of the LWAC layer, the C-ITZ phase is generated by copying and moving the two side boundaries of the LWAC layer a certain distance outside which equals to the thickness of the C-ITZ. The experiment is designed that both sides of the NWC layers are cast after the casting interface is handled by the mortar retarder. Therefore, the N-AGG in NWC does not intrude into the C-ITZ in the model. The detail of the multiphase mesoscopic model of the FGC is shown in Fig. 5. 


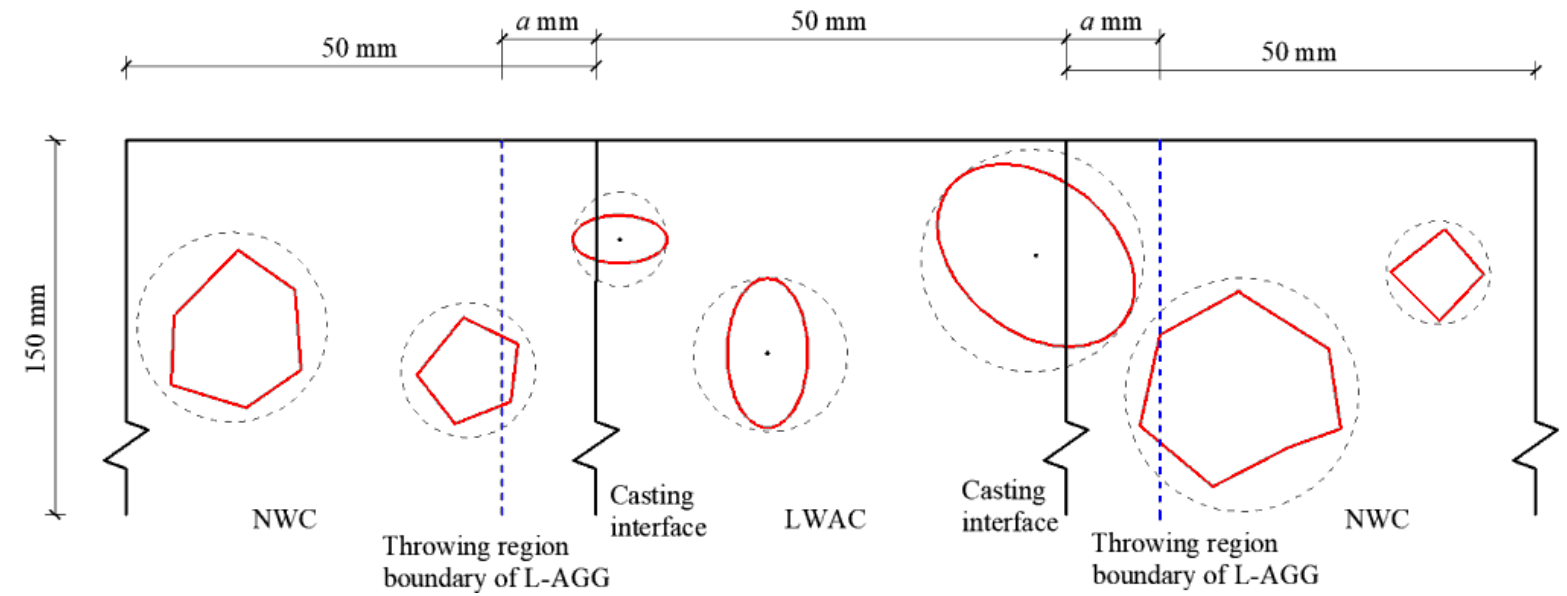

Fig. 4 Positioning scheme of coarse aggregates in the FGC model.

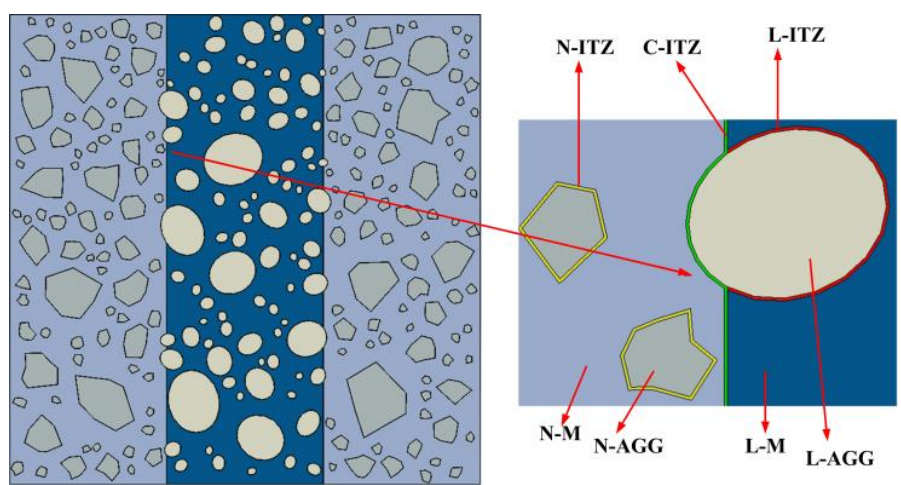

Fig. 5 Multiphase mesoscopic model of FGC.

\subsection{Constitutive model of each phase in FGC}

The constitutive damage plastic model (CDP) is a general model to simulate the concrete performance under cyclic and dynamic loads. It describes the inelastic performance of concrete based on the isotropic damage elasticity together with the isotropic stretching and compression damage plasticity. It is assumed that the concrete is damaged because of the tensile cracking and compression crushing. The necessary parameters to describe the CDP of each phase in FGC are the tensile and compression stress-strain curves, the tensile and compression damage factor-inelastic strain curves. If lacking adequate experimental results, these parameters can be determined according to the stress-strain curve together with the principle of energy equivalence. ${ }^{[17]}$ The tensile and compression stressstrain curves are shown in Fig. 6.

The inelastic strain can be calculated according to the relationship of the parameters in the stress-strain curve as expressed by Equations (3) and (4):

$$
\begin{aligned}
& \varepsilon_{c}^{i n}=\varepsilon_{c}-\varepsilon_{o c}^{e l} ; \quad \varepsilon_{o c}^{i n}=\frac{\sigma_{c}}{E_{o}} ; \quad \varepsilon_{c}^{p l}=\varepsilon_{c}^{i n}-\frac{d}{(1-d)} \frac{\sigma_{c}}{E_{o}} \\
& \varepsilon_{t}^{c k}=\varepsilon_{t}-\varepsilon_{o t}^{e l} ; \quad \varepsilon_{o t}^{e l}=\frac{\sigma_{t}}{E_{o}} ; \quad \varepsilon_{t}^{p l}=\varepsilon_{t}^{c k}-\frac{d}{(1-d)} \frac{\sigma_{t}}{E_{o}}
\end{aligned}
$$

in which $\varepsilon_{c}^{i n}$ and $\varepsilon_{t}^{c k}$ are the inelastic strain of the compression and tension respectively; $\varepsilon_{\mathrm{c}}$ and $\varepsilon_{\mathrm{t}}$ are the actual compressive and tensile strain; $\varepsilon_{o c}^{i n}$ and $\varepsilon_{o t}^{e l}$ are the elastic strain in compression and tension at the initial stiffness level; and $d$ is the damage factor representing the degradation of the elastic stiffness of concrete. Its value varies from 0 (nondestructive) to 1 (total loss); $\varepsilon_{c}^{p l}$ and $\varepsilon_{t}^{p l}$ are the plastic strain in compression and tension, and their values should be greater than 0 and increase with the value of $\varepsilon_{c}\left(\varepsilon_{t}\right)$ to indicate that the concrete enters the plastic softening stage.

The fracture energy is a substantial material property to measure the ability of materials to resist the damage crack growth. ${ }^{[18]}$ Considering the uncertainness of the parameters influencing the damage representation in the simulation and according to the energy equivalence principle of Sidiroff, ${ }^{[19]}$ the residual elastic energy generated by the stress acting on the damaged material equals to that in the non-destructive material. Thus, the damage factor of concrete can be expressed by Equation (5),

$$
d=1-\sqrt{\frac{\sigma}{\left(E_{\mathrm{o}} \varepsilon\right)}}
$$

where $d$ is the damage factor, $\sigma$ is the true stress, $\varepsilon$ is the real strain, and $E_{\mathrm{o}}$ is the initial elastic modulus.

\subsection{Mechanical parameters of phases in FGC}

There are seven kinds of internal phases needed to be defined for the multiphase mesoscopic model: N-AGG, N-M, N-ITZ, L-AGG, L-M, L-ITZ and C-ITZ. The compressive and tensile strengths of N-AGG can be obtained by the point loading test of the rock. ${ }^{[2]}$ The elastic modulus and tensile strength of LAGG can be determined according to European codes and tests. ${ }^{[21]}$ The tensile strength of L-AGG is about $1 / 8$ of the compressive strength, and it increases with increasing the particle density of the L-AGG. The elastic modulus of L-AGG is determined by Equation (6),

$$
E_{\mathrm{LC}}=0.008 \rho_{a}^{2}
$$

where $\rho_{\mathrm{a}}$ is the apparent density of L-AGG. 

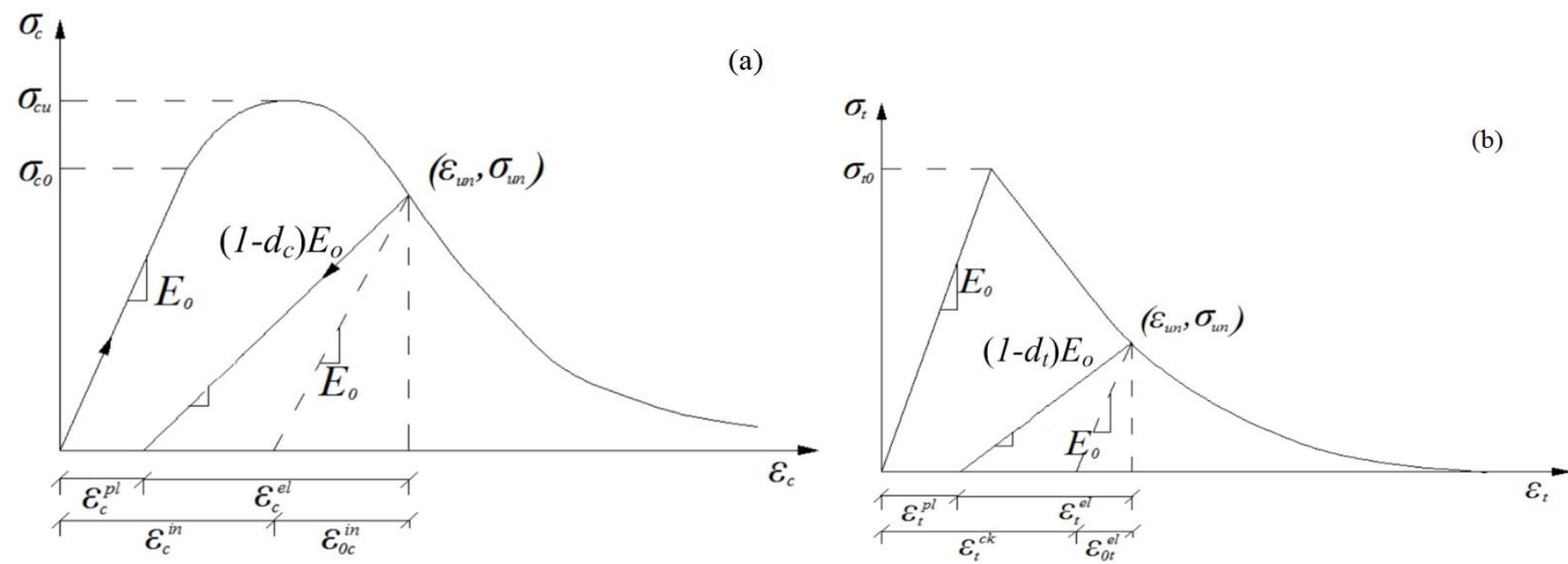

Fig. 6 Uniaxial stress-strain relationship curves and inelastic strain of concrete ((a) Diagram of compression stress-strain relationship and inelastic strain $\varepsilon_{c}^{i n}$; and (b) Diagram of tension stress-strain relationship and inelastic strain $\varepsilon_{t}^{c k}$ ).

The parameters of the mortar substrate in concrete can be determined from the relevant literature, ${ }^{[22]}$ and the compressive and tensile strengths can be obtained from the following empirical formula (Equations (7\&8)) according to the watercement ratio in the mortar,

$$
\begin{gathered}
f_{\mathrm{tm}}=1.4 \operatorname{Ln}\left(f_{\mathrm{cm}}\right)-1.5 \\
c / w=0.047 f_{\mathrm{cm}}+0.5
\end{gathered}
$$

in which $f_{\mathrm{cm}}$ and $f_{\mathrm{tm}}$ are the compressive and tensile strengths of the mortar respectively, $c / w$ is the water-cement ratio.

ITZ is considered as a kind of mortar matrix substrate material with higher porosity, and its mechanical properties can be characterized by the weakened mortar matrix material. ${ }^{[23]}$ It indicates that the strength of N-ITZ is among $40 \%$ and $80 \%$ that of the mortar. ${ }^{[2]}$ Due to the internal porous characteristics of the ceramsite, the internal water releases in the later hydration stage. These factors can increase the strength of the surrounding L-ITZ to a certain extent. At the same time, the rough surface of the L-AGG will enhance the engagement with L-ITZ. Therefore, the strength of L-ITZ is higher than that of N-ITZ. In addition, other parameters of N-
ITZ and L-ITZ are the same as those of the N-M and LC-M respectively.

Research shows that C-ITZ is the weak link in the FGC, and the failure mode of the shear specimen breaks along the casting interface. If the hardened LWAC layer is assumed as a super large aggregate, the C-ITZ can be considered as a kind of special ITZ ${ }^{[24]}$ formed on the surface of the super large aggregate. Therefore, the property of the C-ITZ can be characterized by that of the weakened N-M material referring to the related calculation method of the strength of N-ITZ. ${ }^{[23]}$ At last, the property parameters of each phase in the FGC model are listed in Table 1.

\subsection{Boundary condition setting of the model and solving}

Being consistent with the experimental loading scheme shown in Fig.1(b), the local rigid boundary condition is set at the supports and the loading point to avoid the local stress concentration during computing. The displacement loading method is used to control the computing; and the explicit dynamic FEM analysis method is adopted to simulate the bonding performance of the designed FGC model under the shear loading.

\begin{tabular}{|c|c|c|c|c|c|c|c|c|c|}
\hline Name & $\begin{array}{c}\text { Density } \rho \\
\left(\mathbf{k g} / \mathbf{m}^{3}\right)\end{array}$ & $\begin{array}{c}\text { Elastic } \\
\text { modulus } \\
E / G P a \\
\end{array}$ & $\begin{array}{c}\text { Poisson's } \\
\text { ratio } \\
y \\
\end{array}$ & $\begin{array}{c}\text { Shear } \\
\text { angle } \\
\psi\left({ }^{\circ}\right) \\
\end{array}$ & $\begin{array}{c}\text { Eccentricity } \\
\eta(\%)\end{array}$ & $\begin{array}{c}\text { Stress } \\
\text { ratio } \\
f_{\text {bo }} / f_{\text {co }} \\
\end{array}$ & $\mathbf{K}$ & $\begin{array}{c}\text { Compressive } \\
\text { strength } \\
\sigma_{c} / \mathrm{MPa} \\
\end{array}$ & $\begin{array}{c}\text { Tensile } \\
\text { strength } \\
\sigma_{t} / \mathrm{MPa} \\
\end{array}$ \\
\hline N-AGG & $* 2880$ & $* 50$ & $* 0.16$ & $* 30$ & $* 0.1$ & $* 1.16$ & $* 0.67$ & $* 80$ & $* 6$ \\
\hline N-M & $* 2100$ & $* 30$ & $* 0.20$ & $* 30$ & $* 0.1$ & $* 1.16$ & $* 0.67$ & *32.98 & $* 3.4$ \\
\hline N-ITZ & $* 2100$ & $* 25$ & $* 0.20$ & $* 30$ & $* 0.1$ & $* 1.16$ & $* 0.67$ & $* 24.74$ & $* 2.99$ \\
\hline L-AGG & \& 1796 & $* 25.8$ & $* 0.18$ & $* 30$ & $* 0.1$ & $* 1.16$ & $* 0.67$ & ${ }^{\#} 22.4$ & $* 2.8$ \\
\hline L-M & $* 2100$ & $* 31$ & $* 0.20$ & $* 30$ & $* 0.1$ & *1.16 & $* 0.67$ & *36.93 & $* 3.55$ \\
\hline L-ITZ & $* 2100$ & $* 28$ & $* 0.20$ & $* 30$ & $* 0.1$ & $* 1.16$ & $* 0.67$ & \#29.54 & \#3.24 \\
\hline C-ITZ & $* 2100$ & \#23 & $* 0.20$ & $* 30$ & $* 0.1$ & $* 1.16$ & $* 0.67$ & ${ }^{\# 1} 19.79$ & $\#_{2.68}$ \\
\hline
\end{tabular}

Table 1. Mechanical parameters of each phase in the FGC model.

* from the relevant reference, " from the trial simulation, ${ }^{*}$ from the experimental test. 


\section{Results and discussion}

\subsection{Experiment and the verification of the model}

A series of sandwich FGC experimental blocks of $150 \mathrm{~mm} \times 150 \mathrm{~mm} \times 150 \mathrm{~mm}$ dimensions were made with the LWAC layer as the middle substrate cast by NWC on both sides. Three casting interval time, 10 hours, 2 days and 28 days, were considered in the experiment; and the casting interface was handled by the mortar retarder. The mixture ratio of LWAC and NWC is listed in Table 2. The stacking and apparent densities of the ellipsoidal ceramsite aggregate are 988 and $1796 \mathrm{~kg} / \mathrm{m}^{3}$, respectively, and the cylindrical compression strength of the ceramsite aggregate is $8.4 \mathrm{MPa}$. The typical appearance of the casting interface after handled with the mortar retarder is shown in Fig.2 (a).

The experimental blocks were taken out in time for the shear loading test as shown in Fig.1(b) after 28 days of standard curing saturated in water; and the loading speed was $6 \mathrm{kN} / \mathrm{sec}$. The experimental blocks were finally damaged characterizing with typical brittle shear failure. The final representative failure state of the blocks is shown in Fig.7. The shear damage interface is characterized with clear adhesion between the LWAC middle layer and the NWC layer with the casting interval time being 10 hours. Moreover, this adhesive internal damage in the LWAC layer is obvious than that in NWC layers on both sides. It is due to the weaker strength of the LWAC and the ceramsite aggregates in it compared to that of the NWC and the crushed stones. As the casting interval time extending, this adhesive damage characteristic is weaker. For the case with the casting interval time being 28 days, the shear damage cracks of the blocks were typical smooth as shown in Fig.7(c). At the same time, there is a clear decrease in the final shear loading capacity for the experimental blocks with the increase in casting interval time.

To simulate the influence of the casting interval time on the performance of the FGC blocks, the related main parameters of the C-ITZ in the model were determined by a series of gradual simulation trials and analyses based on the experimental results. The obtained parameters are listed in Table 3.

For the modeling verification and comparison with the experiment, the relative shear displacement of the middle LWAC layer to the NWC layers on both sides of the experimental blocks with the casting interval time being 2 days is extracted and shown in Fig.8. The result from the simulation matches well on the whole with the experimental results. The shear displacement variation with regard to the loading from the simulation shows a typically linear characteristic until to the elastic limit. Subsequently, the FGC performs with the internal damage crack emerging and being developed. During this stage, the load-displacement curve changes into unsmooth as the load increases due to the uneven internal damage happening. It is not until the ultimate load state that the internal crack damage develops very quickly.

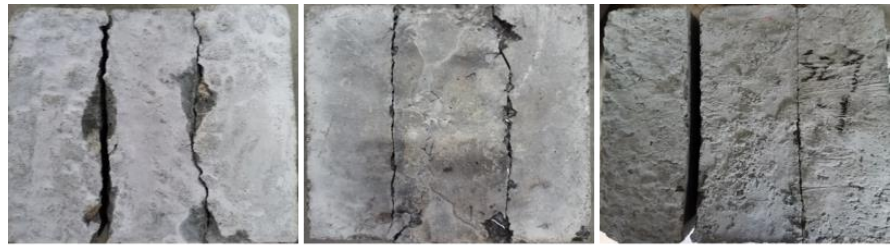

(a) 10 hours

(b) 2 days

(c) 28 days

Fig. 7 Final failure state of Sandwich blocks under shear loading with different casting interval times.

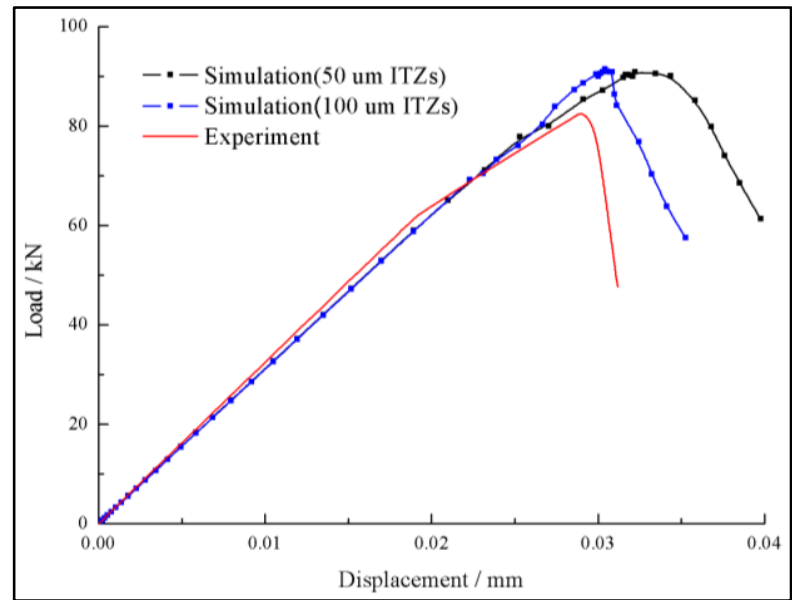

Fig. 8 Relative shear displacement relationship of the FGC specimen blocks and model.

\subsection{Simulation results considering different casting interval time}

Corresponding to the experimental grouping, the model mesh and simulation results are shown in Fig. 9 for the casting interval time being 10 hours, 2 days and 28 days, respectively. The physical structure characteristic of the C-ITZ is reflected in detail in the randomly aggregates model shown in Fig.9(a). The internal damage can be reflected by the plastic strain parameter of the CDP model. Fig. 9(b)-Fig. 9(e) shows the developing of the internal damage in the FGC model with regarding to the shear loading.

There is no internal damage emerging in the model during the loading beginning state. The FGC performs with typically linear elastic characteristics; and the C-ITZ deforms mainly with the shear characteristic. For all cases with different casting interval times in modeling, the internal damage first emerges locally along the C-ITZ shown in Fig. 9(b). When the damage in the C-ITZ develops to meet the exposed ceramsite aggregate particle on the casting interface, the ceramsite is damaged due to its lower strength as the load increases as shown in Fig. 9(c). But this state is different for different casting interval time. Because of the excellent bonding performance of the C-ITZ with a shorter casting interval time being only 10 hours, the main mechanical parameters of the C-ITZ in the model, the elastic modulus, the compressive strength and the tensile strength are higher than those of LAGG exposed on the C-ITZ. The internal damage in the exposed cermasite aggregates on the C-ITZ is more distinct than the other two cases. Comparatively, for the casting 
interval time being 28 days, the mechanical property of the C- the obvious damage in LWAC near the C-ITZ. Moreover, the ITZ listed in Table3 is much weaker than that of the L-AGG appearance of the damaged surface is very rough. in the model. Therefore, as the damage in the C-ITZ develops Comparatively, the final damaged surface of the model for the meeting the exposed ceramsite aggregates, the damage in the L-AGG is less obvious than that happened in cases with the casting interval time being 10 hours or 2 days. This difference is shown in Fig. 9(c) and Fig. 9(d). Finally, the failure state of the model with the casting interval time being 10 hours is characterized with both the shear damage on the C-ITZ and casting interval time being 28 days is much smoother shown in Fig. 9(e), which is coincided with that from the experiment shown in Fig. 7(c). There are parts of exposed ceramsite aggregates on the C-ITZ with slighter damage compared with that of the casting interval time being only 10 hours.

Table 2. Mixture ratio of LWAC and NWC of the experiment.

\begin{tabular}{|c|c|c|c|c|c|c|c|}
\hline \multirow{2}{*}{$\begin{array}{c}\text { Strength } \\
\text { grade }\end{array}$} & \multicolumn{6}{|c|}{$\left(\mathrm{kg} / \mathrm{m}^{3}\right)$} & \multirow{2}{*}{$\begin{array}{c}\text { Compressive strength } \\
\text { (28 days, } \mathrm{MPa})\end{array}$} \\
\hline & Ceramsite & Crushed stone & Sand & Cement & Fly ash & Water & \\
\hline LC30 & 728 & 1 & 495 & 450 & 80 & 237 & 31.0 \\
\hline C40 & I & 1200 & 591 & 410 & 80 & 200 & 48.9 \\
\hline
\end{tabular}

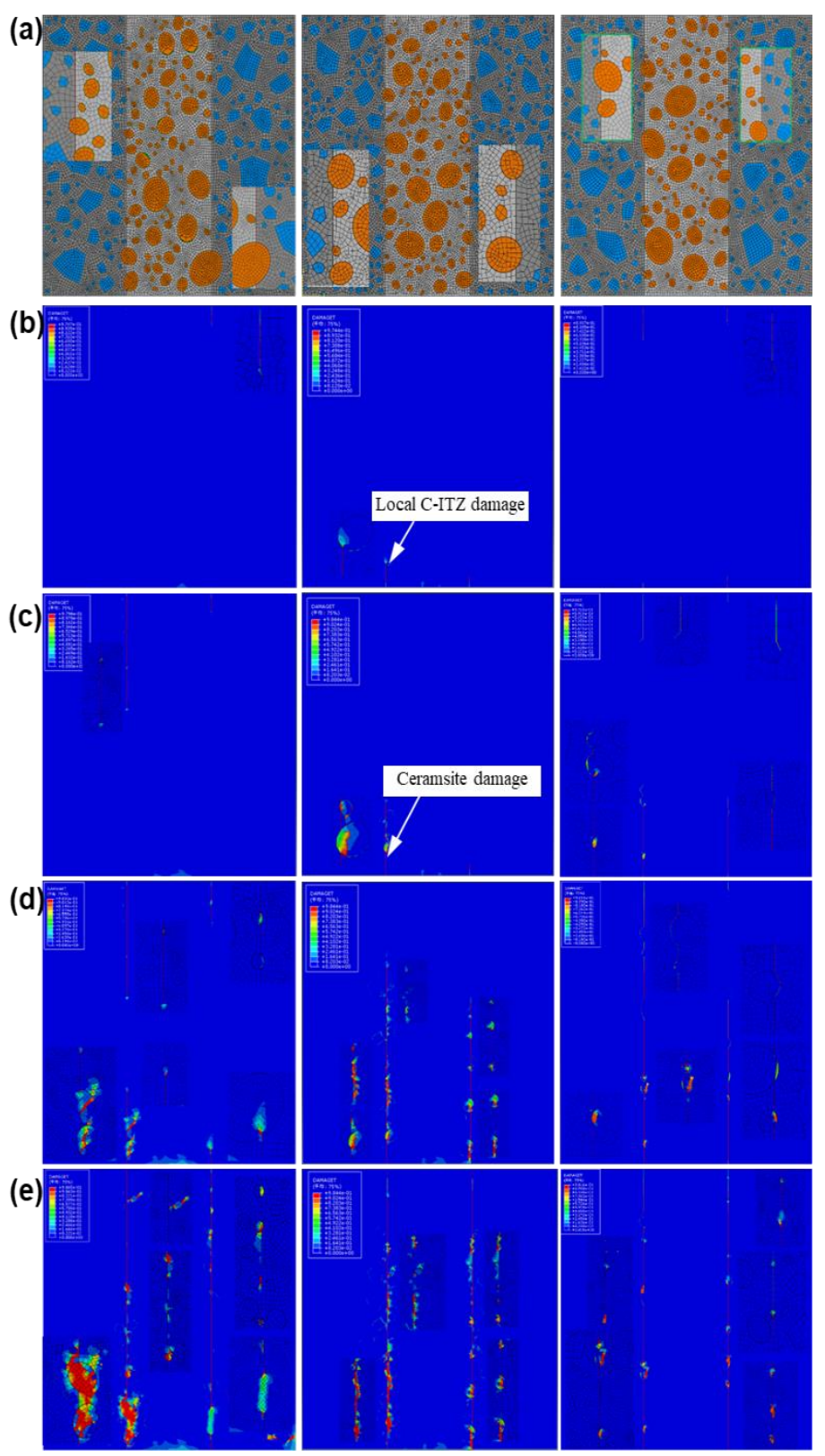

Fig. 9 Model mesh and the internal damage developing for different casting interval times [(a) Mesh of the random aggregate model, (b) Internal damage emerging, (c) Damage developing, (d) Ultimate loading state, and (e) Final failure state]. 


\subsection{Influence of the thickness of ITZs in modeling}

Different thicknesses of the ITZs and mesh in modeling were considered to qualitatively study the influence on the simulation. Fig. 10 shows the results of the loading process with the distribution of the same aggregates but with different thicknesses of the ITZs in the model. Under the same simulating loading scheme, the ultimate loads for thicknesses of 50 and $100 \mu \mathrm{m}$ are 90.8 and $91.4 \mathrm{kN}$, respectively as shown in Fig. 8; and the corresponding relative slip displacements are about 0.032 and $0.031 \mathrm{~mm}$, respectively. The ultimate load corresponding to the experiment is $82.8 \mathrm{kN}$.

It can be observed from Fig. 8 that the load-displacement relationship shows an identical coincidence before the internal damage emerging in both models with different thicknesses of the ITZs. For the approximately equal displacement loading steps, there is no obvious difference in the emergence of the initial internal damage on the distribution and the position in the model. Because of the same distribution of aggregates in the model, the damage state of the ultimate load is identical except a slight difference in the local damage degree. This situation is similar to the final failure state.

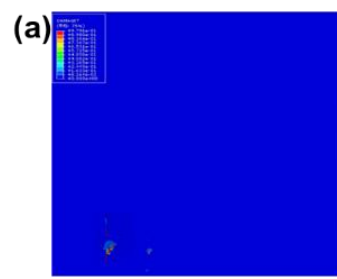

Dis: $0.020 \mathrm{~mm}$

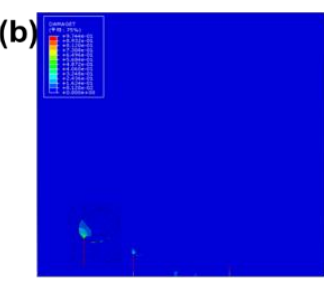

Dis: $0.020 \mathrm{~mm}$

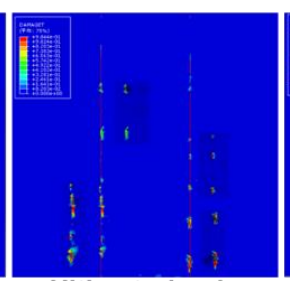

Ultimate load (Dis: $0.032 \mathrm{~mm}$ )

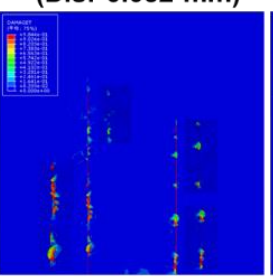

Ultimate load (Dis: $0.032 \mathrm{~mm}$ )

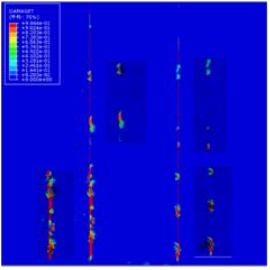

Final damage state

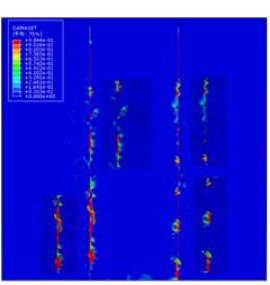

Final damage state
Fig. 10 Results of the model with different thicknesses of the ITZs [(a)50 $\mu \mathrm{m}$, and (b) $100 \mu \mathrm{m}]$.

Table 3. Parameters of the C-ITZ in the model for different casting interval time.

\begin{tabular}{|c|c|c|c|c|c|c|c|c|c|}
\hline $\begin{array}{c}\text { Casting } \\
\text { interval } \\
\text { time } \\
\end{array}$ & $\begin{array}{c}\text { Density } \rho \\
\left(\mathrm{kg} / \mathrm{m}^{3}\right)\end{array}$ & $\begin{array}{c}\text { Elastic } \\
\text { Modulus } \\
\text { E/GPa } \\
\end{array}$ & $\begin{array}{c}\text { Poisson's } \\
\text { ratio } \\
v \\
\end{array}$ & $\begin{array}{c}\text { Shear } \\
\text { angle } \\
\psi(9) \\
\end{array}$ & $\begin{array}{c}\text { Eccentricity } \\
\eta(\%)\end{array}$ & $\begin{array}{l}\text { Stress } \\
\text { ratio } \\
f_{\mathrm{bo}} / f_{\mathrm{co}} \\
\end{array}$ & $\mathbf{K}$ & $\begin{array}{c}\text { Compressive } \\
\text { strength } \\
\sigma_{c} / \mathrm{MPa} \\
\end{array}$ & $\begin{array}{l}\text { Tensile } \\
\text { strength } \\
\sigma_{t} / \mathrm{MPa} \\
\end{array}$ \\
\hline $10 \mathrm{~h}$ & $* 2100$ & ${ }^{\#} 27$ & $* 0.20$ & $* 30$ & $* 0.1$ & ${ }^{*} 1.16$ & ${ }^{*} 0.67$ & ${ }^{\#} 28.03$ & ${ }^{*} 3.17$ \\
\hline $2 \mathrm{~d}$ & $* 2100$ & ${ }^{\#} 23$ & $* 0.20$ & $* 30$ & $* 0.1$ & ${ }^{*} 1.16$ & $*_{0.67}$ & ${ }^{\#} 19.79$ & ${ }^{*} 2.68$ \\
\hline $7 \mathrm{~d}$ & $* 2100$ & ${ }^{\#} 21.5$ & $*_{0.20}$ & $* 30$ & $* 0.1$ & ${ }^{*} 1.16$ & $* 0.67$ & ${ }^{\#} 16.49$ & ${ }^{*} 2.42$ \\
\hline $14 \mathrm{~d}$ & $*_{2} 100$ & ${ }^{\#} 20$ & $* 0.20$ & $* 30$ & $* 0.1$ & *1.16 & $*_{0.67}$ & ${ }^{\#} 11.54$ & ${ }^{\#} 1.92$ \\
\hline $28 \mathrm{~d}$ & $* 2100$ & ${ }^{\#} 18$ & $*_{0.20}$ & $* 30$ & $* 0.1$ & $*^{*} 1.16$ & $* 0.67$ & $\# 8.25$ & ${ }^{\#} 1.45$ \\
\hline
\end{tabular}

\section{Conclusions}

A kind of modeling method of the multiphase mesoscopic finite element simulation was proposed to analyze the bonding performance of the casting interface in the functionally graded concrete (FGC). The designed FGC model is composed of NWC layers cast on the LWAC layer on both sides corresponding to the experiment.

(1) The physical structural characteristic of the casting interfacial transition zone (C-ITZ) is especially considered in the proposed FGC model and is realized in detail in simulation. The C-ITZ is physically characterized by the partial exposure of ceramsite aggregates on the handled casting interface. Considering the physical structural characteristic of the casting interface and the material property, C-ITZ is separately taken out as an independent internal phase to be simulated in the model.

(2) The load-displacement variation under the shear loading from the simulation generally agrees well with that from the experiment. As the main position to bear the shear load, the internal damage first emerges locally in C-ITZ due to the weaker link of the C-ITZ and the lower strength of the exposed ceramsite on the casting interface. For a shorter casting interval time, the results whether from the experiment or the simulation characterize with obviously rough damage on the C-ITZ because of a better bonding performance of the $\mathrm{C}$-ITZ. With the extending of the casting interval time, the damage changes smoother due to the weakening of the bonding performance of the C-ITZ.

(3) At present, there is no clear difference in the simulation considering different thicknesses in the proposed modeling. As the weaker link in the FGC, the bonding performance of the C-ITZ is directly related to the handled method of the casting interface, casting interval time, the strength and distribution of aggregates near or in the C-ITZ. The mechanical performance and physical structure of the C-ITZ need further research during the simulation and experiment considering the microstructure characteristic, the damage crack mechanism and so on. 


\section{Acknowledgments}

This work was supported by National Natural Science Foundation of China [No. 51878360, No. 51778302] and Natural Science Foundation of Zhejiang Province [LY18E080008].

\section{Conflict of Interest}

There is no conflict of interest.

\section{Supporting Information}

Not Applicable.

\section{References}

[1] J. Yang, R. Hai, Y. Dong and K. Wu, J. Chin. Ceram. Soc., 2002, 30(6), 803-806.

[2] Z. Li, Q. Cui and K. Shi, Concr., 2016, 11, 16-20, doi: 10.3969/j.issn.1002-3550.2016.11.005.

[3] J. Yang, Research on physical and mechanical properties of cement-based functionally gradient composite materials, Tongji University, 2003.

[4] J. Peng, Sci. Technol. Vision, 2012, 18, 117-118, doi: CNKI:SUN:KJSJ.0.2012-18-050

[5] Akmaluddin and S. Murtladi, Procedia Eng., 2013, 54, 286298, doi: 10.1016/j.proeng.2013.03.026.

[6] W. Y. Ji, W. W. Li, M. L. Guo and J. Wang, J. Jilin Univ. (Engineering and Technology Edition), 2018, 48(1), 129-136, doi: 10.13229/j.cnki.jdxbgxb20161204.

[7] I. Monetto and F. Campi, Eng. Struct., 2017, 144, 201-209, doi: 10.1016/j.engstruct.2017.04.010.

[8] F. Campi, and I. Monetto, Int. J. Solids Struct., 2013, 50(5), 687-698, doi: 10.1016/j.ijsolstr.2012.10.032.

[9] F. Dong, Z. Liu and M. Jia, Sichuan Build. Mater., 2018, 44(11), 5-6.

[10] Z. Yao, D. Duan, F. Dang and F. Zhang, J. Xi'an Univ. Technol., 2018, 34(04), 101-106, doi: 10.19322/j.cnki.issn.10064710.2018.04.015.

[11] J. Qu, Z. Lou and Z. Liu, J. Yangtze River Sci. Res. Institute, 2015, 32(4), 129-132, doi: 10.3969/j.issn.10015485.2015.04.025.

[12] Z. Zhang, X. Song, Y. Liu, D. Wu and C. Song, Compos. Sci. Technol., 2017, 149, 235-245, doi: 10.1016/j.compscitech.2017.06.015.

[13] J. C. Walraven, Delft University Press, 1980, 67-72.

[14] H. Shi, D. Sun and K. Wu, J. Chin. Ceram. Soc., 2016, 44(5), 678-685, doi: 10.14062/j.issn.0454-5648.2016.05.10.

[15] K. Liao, P. Chang, Y. Peng and C. Yang, Cem. Concr. Res., 2004, 34, 977-989, doi: 10.1016/j.cemconres.2003.11.019.

[16] X. Du and L. Jin, Eng. Mech., 2012, 29(12), 72-79, doi: 10.6052/j.issn.1000-4750.2011.04.0216.

[17] Code for Design of Concrete Structures, GB50010-2010.

[18] K. M. Hamdia, M. Silani, X. Y. Zhuang, P. F. He and T. Rabczuk, Int. J. Fracture, 2017, 206(2), 215-227, doi: 10.1007/s10704-017-0210-6.

[19] Z. Li, Damage mechanics and its application. Beijing: Science Press, 2002.

[20] L. Sun, Agric. Sci. Technol., 2012, 14, 50-51, doi: 10.3969/j.issn.1003-6997.2012.14.034.

[21] J. Liu and C. Zhang, Sci. Technol. Eng., 2018, 18(18), 269274.

[22] G. Chen. Mesoscopic simulation of mechanical properties for lightweight aggregate concrete [D]. Dalian University of Technology, 2007.

[23] M. Du, L. Jin, D. Li and X. Du, Eng. Mech., 2017, 34(9), 5463, doi: 10.6052/j.issn.1000-4750.2016.02.0122.

[24] C. Hu and M. Wu, Shanxi Architect., 2012, 38(1), 116-117, doi: 10.3969/j.issn.1009-6825.2012.01.068.

Publisher's Note Engineered Science Publisher remains neutral with regard to jurisdictional claims in published maps and institutional affiliations. 